\title{
Electrostatic protection analysis of the pasting equipment
}

\author{
Minghui You ${ }^{1}$, Xinyu $\mathrm{Yu}^{2}$, Qixiang Sun ${ }^{1}$, Liping $\mathrm{Yin}^{1}$,Shijun $\mathrm{LI}^{1}$, \\ Xue $\mathrm{Li}^{1}$, Jingsheng Liu* ${ }^{1, \text { a }}$ \\ ${ }^{1}$ Jilin Agricultural University, Changchun 130118, Jilin, China \\ ${ }^{2}$ Jilin Institute of agricultural machinery, Changchun 130021, Jilin, China \\ *mhyou000@163.com
}

Keywords: static electric field, electrostatic protection, electrostatic shielding

\begin{abstract}
In this paper, we introduced the theory of static electricity protection for heating and pasting equipment. Conductors were electrically conductive objects, in which there were free to move the charge; we studied the metal conductor, which was characterized by the existence of lots of free movement of internal electrons. Due to the characteristics of the metal conductor in the electric field, the role of electric field would be force the free electrons to move in the conductor surface charge redistribution phenomenon. When there was no charge in the conductor for the state of the static movement of static equilibrium. The static equilibrium states and conditions were analyzed theoretically, respectively.
\end{abstract}

\section{Introduction}

In the living environment, the vacuum was only an ideal situation, and the actual electric field there would always be some material, from the conductive material into conductors, semiconductors and dielectrics, these substances into the electric field, and the electric field Have interaction and influence. The charge that appeared on the surface was called the induced charge. Electrostatic induction process was non-equilibrium state[1-4]. There was no charge in the conductor for the state of static movement of static equilibrium. Conductor surface charge in two cases: no charge in the plane, there were equal surface charge of different numbers. Suppose there were equal numbers in the plane Charge, then there were an electric field line from the positive charge at the negative charge, we knew that along the direction of the electric field line potential was reduced. Therefore, the potential of the positive charge distribution was higher than that of the negative charge distribution, which was contradictory to the equipotential surface of the conductor surface in electrostatic equilibrium. Therefore, charge was distributed only on the outer surface, and no charge was present on the inner surface[5-7].

Reduce the direct friction of objects, due to avoid frequent contact between objects or separation (including surface stripping and flammable liquid flow) as far as possible. Kept objects clean and free from static electricity. Metal shields were to be applied to the live conductor or its insulation. For machines, equipment, machine grounding is to prevent the best way to generate static electricity. 


\section{Electrostatic induction phenomenon}
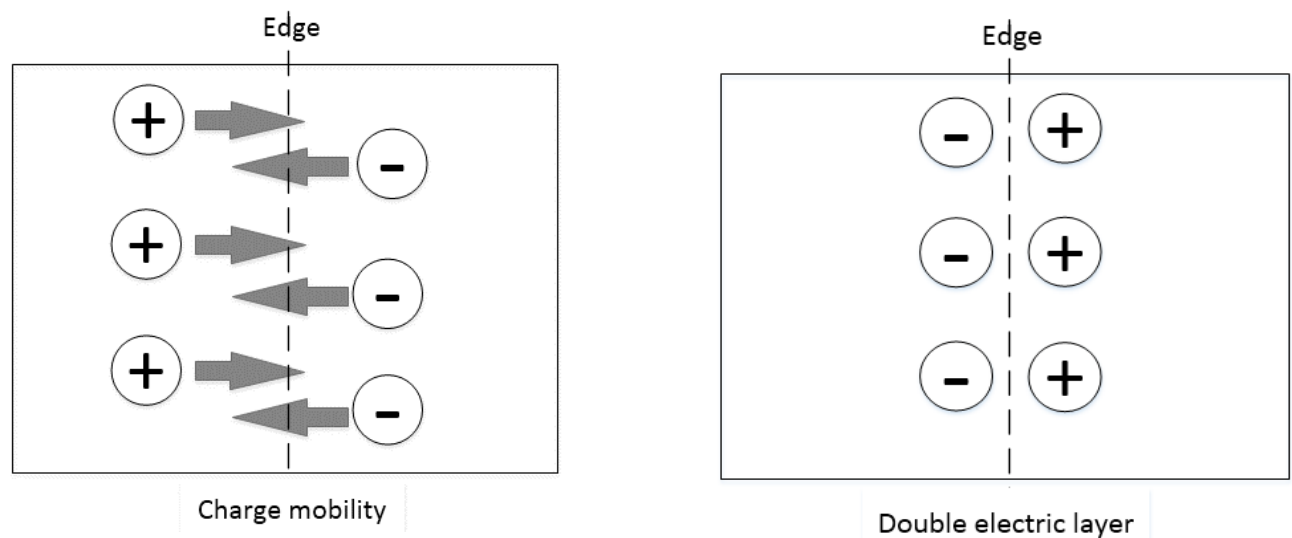

Fig1. Generate static electricity
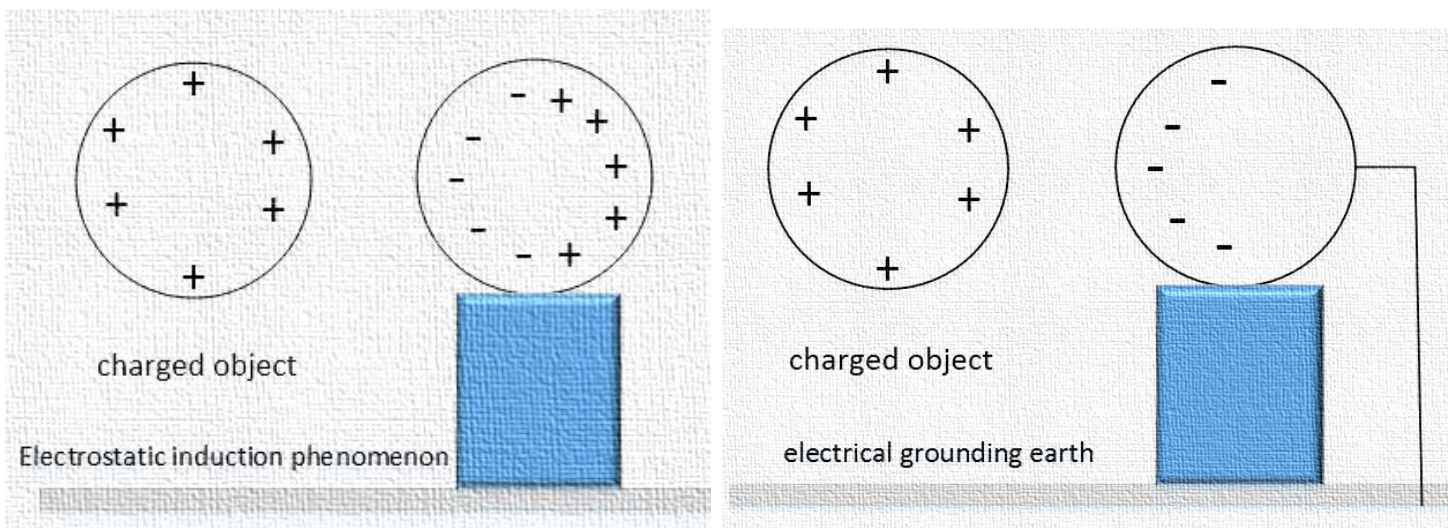

Fig2.Electrostatic induction performance charge and elimination method

\section{Electrostatic shielding and Cavity conductor}

When there was no charged body in the cavity, the electric charge of the conductor was distributed only on its outer surface. In the conductor inside the arbitrary take a closed curved surface S, because the electric field strength inside the conductor was zero, by Gauss theorem.

$$
\oint_{S} \vec{E} \cdot d \vec{S}=\frac{1}{\varepsilon_{0}} \sum_{i} q_{i}=0
$$

So the conductor surface charge in two cases: no charge in the face, The same number of different surface charge. Suppose there were equal numbers in the plane Charge, then there must be an electric field line from the positive charge at the negative charge, we know that along the direction of the electric field line potential was reduced. Therefore, the potential of the positive charge distribution was higher than that of the negative charge distribution, which was contradictory to the equipotential surface of the conductor surface in electrostatic equilibrium. Therefore, charge was distributed only on the outer surface, and no charge was present on the inner surface.

There was no charge on the inner surface. The relationship between the surface charge density and the field strength means that no electric field in the immediate vicinity of the conductor surface. If there was an electric field in the internal space, there will be a distribution of the electric field lines, which must be interrupted. Not only in the absence of charge to interrupt the contradiction, but also the entire conductor inside the space electric field strength is zero everywhere.

In the cavity part of the optional point $\mathrm{A}$, the cavity part of the optional point $\mathrm{B}$, calculate the 
potential difference between $\mathrm{AB}$ two points, so the two potential difference is zero. The two points are optional, so that the cavity portion and the cavity portion are all an equipotential body. Electrostatic shielding: The internal electric field of a cavity conductor (whether grounded or not) was not affected by an external electric field and can shield the external field from internal influences.

When there was a charged body in the cavity, the algebraic sum of charge and cavity charge in the inner surface of the cavity conductor was zero.Cavity charge $+q$, then there will certainly be on the surface of the negative charge distribution, how much charge power? Still using the Gaussian theorem, in the cavity part of a closed Gaussian surface. Because the left side was zero, so the inner surface with the same number of different charges, the charge on the inner surface of the charge and the charge of algebraic sum of zero, according to the conservation of charge, the outer surface charge $+q$.

When the change in the cavity charge charge, the external surface charge changes, so this time the internal charge on the field have an impact. However, if we ground the outer surface, the external field will be no longer affect if we change the charge or position of the internal charge anyway. A grounded cavity conductor shields the external field from internal influences and also shields the internal field from external fields. This phenomenon was called electrostatic shielding.

\section{Conclusion}

When the change in the cavity was charged, the external surface would be changes. At same time, the internal charge on the field had an impact. However, if we ground the outer surface, the external field will no longer be affected, if it will be changed the charge or position of the internal charge anyway. Not only grounding the conductor could shield the external field of internal impact, but also shield the internal field of the impact of external field, it could play a static electricity protection equipment role.

\section{Acknowledgments}

The authors will thanks gratitude to the projects: Jilin Province Education Department Project (No. 2015Y174) , Jilin Agricultural University learning program , Jilin Provincial Department of human resources and social security Project (No. 2015Y24)for their generous support of this work.

\section{References}

[1]Boeing Company 737-600/700/800/900, AIRCRAFT MAINTENANCE MANUAL, USA : Boein (2012).

[2]Han F., Gao Z., Li D., et al. Nonlinear Compensation of Active Electrostatic Bearings Supporting a Spherical Rotor. Sensors and Actuators A: Physical, 2005, 119(1): 177-186.

[3]C.J.Sletten.Electromagnetic Scattering from Wedges and Cones,CambridgeResearch Center,Report CRC-E5090,July 2002

[4]J.J Bowman.T.B.A.Senior and P.L.E.Uslenghi,Electromagnetic and AcousticScattering by Simple Shapes,Amsterdam: North-Holl and PublishingCo.,2009

[5]Clayton $\mathrm{R}$ P,Leighton $\mathrm{R}$ B,Sands M.The feyman lectures on physics (VolumeII )[M].[s.l.]:Pearson Education Inc.Publication,1989.

[6]Sullivan D M.Electromagnetic simulation using the FDTD method .New York Press,2000.

[7]M.Li,K.P.Ma,D.M.Hockanson,etc..Numerical experimental Corroboration of an FDTD thin-slot model for slots near of Shielding enclosures. IEEE Trans Electromagnetic compatible corners,vo139,no3,1997,225-232. 